\title{
The Allocated Maximal Backup Covering Tour Problem with Minimum Building Cost
}

\author{
Rashed Sahraeian (Corresponding author) \\ Assistant Professor of Industrial Engineering \\ Shahed University, Tehran, Iran \\ E-mail: sahraeian@shahed.ac.ir
}

Ali Ebrahimi

Master of Science Student, Industrial Engineering Department

Shahed University, Tehran, Iran

E-mail: aliebrahimi@shahed.ac.ir

\author{
Received: November 1, $2011 \quad$ Accepted: November 21, $2011 \quad$ Published: March 1, 2012 \\ doi:10.5539/jms.v2n1p151 URL: http://dx.doi.org/10.5539/jms.v2n1p151
}

\begin{abstract}
This study addresses the Allocated Maximal Backup Covering Tour Problem (AMBCTP) that is a generalization of the Covering Tour Problem (CTP). This problem is defined on an undirected graph $G=(V \cup W, E)$, where $W$ is a set of vertices that must be collectively covered by a vehicle. The AMBCTP consist of determining a minimum length vehicle route on a subset of $V$, subject to side constraints, such that every vertex of $W$ is within a pre-specified distance from the route. Maximizing number of vertices of $W$ set which are covered for second or more times is another objective in this problem. Moreover, allocation cost of the every vertex of $W$ to one vertex of $V$ which is belonging to the tour is minimized. Transmission vehicle from each city, in health care teams example that provided by Current and Schilling (1994) for this problem, requires to build a clinic in it, therefore we considered a fixed and variable cost (i.e. building cost) for visited cities, that must be minimized. Mathematical formulation of the AMBCTP, that is a multi-objective problem, is proposed. We used a powerful Multi-objective Decision Making (MODM) method for optimizing it. Finally a numerical example is provided to demonstrate the validity of the model.
\end{abstract}

Keywords: The allocated maximal backup covering tour problem, Multi-objective decision making, Global criterion method

\section{Introduction}

This paper represents an extension of the CTP (that is a combination of Traveling Salesman Problem and Set Covering Problem), namely Allocated Maximal Backup Covering Tour Problem. Since the CTP is a NP-hard problem, so the AMBCTP also will be NP-hard. The AMBCTP is a multi-objective problem and aims to determine a minimal length tour for a subset of nodes ( $V$ set) while also maximizing number of vertices in set of $W$ which is covered for second or more times by a node of $V$ set which is belonging to the tour, i.e. maximizing backup coverage. Moreover, allocation cost of the every vertex of $W$ set to one vertex of $V$ set which is belonging to the tour is minimized. In this goal, number of vertices in $W$ set that is allocated to any vertex of $V$ set, is determined and so vehicle driver can pre-schedule for stop time unites in each visited node. Note that allocation cost is in the distance form. An example provided by Current and Schilling (1994) for this problem is construction of a route for visiting health care teams in developing countries, where medical services can only be delivered to a subset of cities, but all users must be able to reach a visiting medical team, so transmission vehicle from each city, in this case, requires to build a clinic in it, therefore we considered a fixed and variable cost (i.e. building cost) for visited cities, that must be minimized.

Some when, some of nature incidents such as flood and earthquake cause that people reaching to selected locations be impossible, also it may be required in high-demand areas to maintain a uniform level of service when vehicles can respond to only one call at a time (Hogan \& revelle, 1986), so it is necessary that backup 
coverage in selecting of locations for people welfare (or set of W) is considered. In this example, that we defined the AMBCTP on it, nodes of $\mathrm{V}$ set refer to cities set and vertices of $\mathrm{W}$ set refer to demand points that need to medical services.

The AMBCTP can be formally described as follows: let $G=(V \cup W, E)$ be an undirected graph, where $V \bigcup W$ is the vertex set, and $E=\left\{\left(v_{i}, v_{j}\right) \mid v_{i}, v_{j} \in V \bigcup W, i<j\right\}$ is the edge set. Vertex $v_{1}$ is a depot, $\mathrm{V}$ is the set of vertices that can be visited, $|V|=n, \mathrm{~T} \subseteq \mathrm{V}$ is the set of vertices that must be visited ( $v_{1} \in T$ ), and $\mathrm{W}$ is the set of vertices that must be covered. A distance matrix $C=\left(c_{i j}\right)$, satisfying triangle inequality, is defined for $\mathrm{E}$. Another distance matrix $D=\left(d_{l k}\right)$, also is defined for distance between $v_{l} \in W$ and $v_{k} \in V$. Vectors $F=f_{k}$ and $H=h_{k}$ are associated with fixed and variable clinic building cost, respectively. The AMBCTP consists of defining a tour for a subset of $\mathrm{V}$, which contains all the vertices from $\mathrm{T}$, while at the same time optimizing the following four objectives: (i) the minimization of the tour length, (ii) the maximization of the backup coverage, (iii) the minimization of the allocation cost and finally (iv) the minimization of the total clinic building cost. (ii) and (iii) objectives are known as service level indicators in the AMBCTP in this paper. A feasible solution for a small instance is provided in Figure 1.

As mentioned above the AMBCTP is a generalization of the CTP and though the CTP has not received much attention in literature (Gendreau et. al, 1997), it seems that this problem was first introduced by Current (1981). It is formulated in Current and Schilling (1989). In this reference and in Current and Schilling (1994), a two-objective version of the problem is considered: the length of the tour and the number of vertices covered by the tour. Finally this problem proposed by Gendreau et. al (1997) and an exact branch-and-cut algorithm developed for it and the latter has been applied successfully to the routing of a mobile medical facility in Ghana (Hodgson et. al, 1998). In addition, Maniezzo et. al (1999) have presented a model and three scatter-search algorithms, and Motta et. al (2001) proposed a generalized version of the CTP, namely the Generalized Covering Tour Problem (GCTP), and presented a set of reduction rules, heuristic algorithms and a ILP based formulation for the GCTP, note that Motta et. al (2010) proposed this problem for first time. A bi-objective model of the CTP (BOCTP) formulated by Jozefowiez et. al (2007) that aims to determine a minimal length tour for a subset of nodes while also minimizing the greatest distance between the nodes of another set and the nearest visited node. Hachicha et. al (2000) considered multi-vehicle covering tour problem (m-CTP) that W is a set of vertices that must collectively be covered by up to m vehicles.

To our knowledge backup coverage, location-allocation and building cost have not considered for the CTP in the literature. The proposed model inherits its formulation from the previous researches in maximal covering and location-allocation models. A part of model is formed by using Hogan and ReVelle's (1986) maximal backup coverage model. Hogan and ReVelle (1986) suggested that backup coverage can be used as a decision criterion or location of emergency service vehicles on a network. They describe backup coverage as the second coverage of a demand zone. They applied backup coverage criterion to Set Covering Location Problem (SCLP) and Maximal Covering Location Problem (MCLP) models. First coverage was traded off against backup coverage in their models. It was shown that backup coverage can be provided without substantial first coverage loss (Araz et. al, 2007). For reaching to (iii) aim, we applied location-allocation concept for our suggested model. Cooper (1963) introduced location-allocation problem for the first time. This problem aims at finding optimum number of new facilities, their locations and the optimal allocation of existing facilities to new ones in such a way that total cost of transportation is minimized. In the AMBCTP, existing facilities are vertex of $\mathrm{W}$ set and new facilities are visited vertex of $\mathrm{V}$ set. Considering fixed and variable building cost is an idea that we created for the CTP in this work.

In this paper a powerful MODM method is provided for solving and demonstrating validity of the model, mean: Global Criterion (GC) method. The GC method is one of the most famous of multi-objective optimization methods. This method is proposed by Salukvadze (1974) and many researchers have used it efficiently for multi-objective problems. For example, Sahidul Islam (2008) has used this method for combine the different objective functions to a single objective function in a multi-objective marketing planning inventory problem. In this work, the optimal solutions with good trade-off for different preferences on objective functions are derived truly from applying this method, and so in this paper we also use this method for integrating objective functions and find the optimal solutions for different weights on objective functions.

\section{Model definition}

The AMBCTP can be formulated as a multi-objective integer non-linear program as follows: for $v_{k} \in V$, let $y_{k}$ be a binary variable equal to 1 if and only if vertex $v_{k}$ belongs to the tour. If $v_{k} \in T$, then $y_{k}$ is necessarily equal to 1 . For $\left(v_{i}, v_{j}\right)$ and $i<j$, let $x_{i j}$ be a binary variable equal to 1 if and only if edge $\left(v_{i}, v_{j}\right)$ belongs 
to the tour. Define binary coefficients $\delta_{l k}$ equal to 1 if and only if $v_{l} \in W$ can be covered by $v_{k} \in V$ in primitive radius (i.e. $c_{l k} \leq c$, where $c$ is primitive coverage radius ), and let $S_{l}=\left\{v_{k} \in V \mid \delta_{l k}=1\right\}$ for every $v_{l} \in W$. Hence assume that $\left|S_{l}\right| \geq 2$ for all $v_{l} \in W$ and that the degenerate tour ( $\left.v_{1}\right)$ is infeasible. Also let binary coefficients $\delta_{l k}^{\prime}$ equal to 1 if and only if $v_{l} \in W$ can be covered by $v_{k} \in V$ in secondary radius (i.e. $c_{l k} \leq d$, where $d$ is secondary coverage radius and $\left.d>c\right)$, and let $S_{l}^{\prime}=\left\{v_{k} \in V \mid \delta_{l k}^{\prime}=1\right\}$ for every $v_{l} \in W$. $u_{l}$ is a binary variable that is associated with backup coverage and equal 1 if and only if $v_{l} \in W$ in primitive or secondary radius covered by a vertex of $v_{k} \in V$ for second or more times and there are a benefit coefficients for every $v_{l} \in W$ as $a_{l}$ that indicates importance of vertices of $W$ set. For allocation $v_{l} \in W$ to a vertex of $v_{k} \in V$, we need to define a binary variable as $z_{l k}$ that equal to 1 if and only if vertex $v_{l} \in W$ allocate to $v_{k} \in V$ and integer variable $A_{k}$ is associated with number of vertex $v_{l} \in W$ that are allocated to vertex $v_{k} \in V$. Then the AMBCTP can be stated as:

$$
\begin{array}{cc}
\text { Min } & \sum_{i=0}^{n-1} \sum_{j=i+1}^{n} c_{i j} x_{i j} \\
\text { Min } & \sum_{v_{k} \in V} c_{k} y_{k} \\
\text { Max } & \sum_{l \in W} a_{l} u_{l} \\
\text { Min } & \sum_{v_{l} \in W} \sum_{v_{k} \in V} d_{l k} z_{l k}
\end{array}
$$

Subject to

$$
\begin{aligned}
& \sum_{v_{k} \in S_{l}^{\prime}} y_{k}-U_{l} \geq 1 \quad \forall l \in W \\
& \sum_{v_{k} \in V} z_{l k}=1 \quad \forall l \in W \\
& z_{l k} \leq \delta_{l k} \quad \forall l \in W, v_{k} \in V \\
& z_{l k} \leq y_{k} \quad \forall l \in W, v_{k} \in V \\
& A_{k}=\sum_{l \in W} z_{l k} \quad \forall v_{k} \in V \\
& \sum_{i=1}^{k} x_{i k}+\sum_{j=k+1}^{n} x_{k j}=2 y_{k} \quad \forall v_{k} \in V \\
& \begin{array}{ll}
\sum_{\substack{v_{i} \in S, v_{j} \in V \backslash S \\
\text { or }}} x_{i j} \geq 2 y_{k} & \forall S \subset V, 2 \leq|S| \leq n-2 \\
v_{j} \in S, v_{i} \in V \backslash S & T \backslash S \neq \phi, v_{k} \in S
\end{array} \\
& v_{j} \in S, v_{i} \in V \backslash S \\
& y_{k}=1 \quad \forall v_{k} \in T \\
& A_{k} \in Z^{+} \quad \forall v_{k} \in V \\
& y_{k} \in\{0,1\} \quad \forall v_{k} \in V \backslash T \\
& x_{i j} \in\{0,1\} \quad \forall i, j \in V, i<j \\
& z_{l k} \in\{0,1\} \quad \forall l \in W, v_{k} \in V \\
& u_{l} \in\{0,1\} \quad \forall l \in W
\end{aligned}
$$


In this formulation, objective (1) minimizes the tour length, objective (2) provides maximum backup coverage, objective (3) aims minimizing total allocation cost and objective (4) minimizes total building cost. Note that objective (2) and (3) are service level indicators in the AMBCTP. Constraints (5) are associated with the backup coverage. Constraints (6) ensure that every vertex of $v_{l} \in W$ allocated only to one vertex of $v_{k} \in V$ which is belonging to the tour (i.e. any demand point allocated only to one city which place on the tour). Constraints (7) and (8) ensure that any vertex of $v_{l} \in W$ can allocated to vertices of $v_{k} \in V$ which are in primitive coverage distance and this vertices must be place on the tour, respectively. Constraints (9) is considered for calculating number of vertices $v_{l} \in W$ which allocated to every vertex of $v_{k} \in V$ Constraints (10) are degree constraints and constraints (11) are connectivity constraints and they force the presence of at least two edges between any set $S$ and $V S$, for every proper subset $S$ of $V$ such that $T \backslash S \neq \phi$ and $S$ contains a vertex $v_{k}$ belonging to the tour. Constraints (12) enforce that every vertex of T belongs to the tour. Finally constraints (13), (14), (15), (16) and (17) set the integrality requirements. Note that in this formulation, we do not allow the case where $x_{1 k}=2$ for some $k$ since this can only happen if the tour $\left(v_{1}, v_{k}, v_{1}\right)$ is feasible. The proposed model is multi-objective. Different objectives are usually in conflict and most of the time no optimal solution can be found for this kind of problems. In such cases the concept of optimality is replaced by non-dominance solutions that also called Pareto optimal solutions and will be describe completely in the next section.

\section{Multi-objective optimization}

Multi-objective programming is a mathematical programming method that can investigate multiple definite objectives. It aims to help the decision-makers obtain a better policy under the constraints on finite resources and conflicting objectives (Low et. al, 2005). Output of MODM problems called Pareto optimal solution (i.e. non-dominated solution). A solution is said to be Pareto optimal if it is not dominated by any other solution in the solution space and the set of all feasible non-dominated solutions is referred to as the Pareto optimal set and solutions in the Pareto optimal set have trade-off with together. The ultimate goal of a multi-objective optimization algorithm is to identify solutions in the Pareto optimal set and determine the best optimal solution from this set by decision-maker (Deb, 2001). Elements of the Pareto optimal set should be have a suitable diversity and a uniform partly distribution until the decision maker be confronted with varied solutions. When this goal is provided the decision maker can select a solution based on his preferences. In the classic multi-objective optimization methods, such as whatever that we used in this paper, the different solutions obtain with considering different preference vectors for objectives. But in many discrete problems, such as our problem, there is no information about this case which preference vectors redound to a Pareto optimal set with good specification (Deb, 2001). Since in this paper we introduced some preference vector based on randomness and our mental perception about the problem, of course quality of these vectors is tested by graphical tools.

In this section, we introduce one of the multiple-objective decision making (MODM) methods, which called Global Criterion method and will apply it to determine Pareto optimal set of the AMBCTP as a multi-objective programming in the next subsection.

\subsection{The global criterion method}

The GC method (Salukvadze, 1974), tries to minimize a distance from the optimal solution. The optimal solution is computed by solving all the problems with only one objective function. The optimal value for the function $f_{i}$ is obtained by solving the problem with only $f_{i}$ as objective function. In this way an optimal vector $f^{*}=\left(f_{1}^{*}, f_{2}^{*}, \ldots, f_{k}^{*}\right)$ is obtained. Now a new problem is solved whose objective function is

$$
f(x)=\left(\sum_{i=1}^{k} w_{i}\left[\frac{f_{i}\left(X^{*}\right)-f_{i}(X)}{\left|f_{i}\left(X^{*}\right)\right|}\right]^{P}\right)^{\frac{1}{P}}, \quad(1 \leq P \leq \infty)
$$

where parameter of $P$ defines the type of distance and when reducing distance between an objective function and its optimal value is very important, this parameter is considered very big and inverse, also vector of $w=\left(w_{1}, w_{2}, \ldots, w_{k}\right)$ shows objectives importance and $\sum^{k} w_{i}=1$. Solving result of such a problem is a non-dominance solution and with changing $P$ and vector of $i=1$ other optimal solutions will be obtained that form Pareto optimal set.

\section{Numerical example and analysis}

Because of limitations, we provide a Pareto optimal set only with 10 members. For demonstrating validity of the 
model, one of these solutions will be explain completely. Moreover, we will show trade-off between objective functions through comparison tow solutions with together. Meanwhile, adequacy of the Pareto optimal set will be shown by draw a graph on this set, see Figurer 2. Note that this figure is drawn only for two objectives, first and second objective. Of course other graphs on the other objectives also prove this issue, but because of some limitations we think that to this extent is sufficient.

To the best of our knowledge, there are no test instances for the CTP available in the literature. Hence an experiment was conducted on a randomly generated instance. Because of the AMBCTP is a NP-hard problem and our purpose in this paper is to solve it by exact methods, so we consider very small size of this problem. One instance of problem with 10 cities (as $V$ set) and with 22 demand points (as $W$ set) using GC method is ran and Pareto optimal solutions for this instance are computed. For applying GC method, first, we shall obtain the optimal value for any objective function of model by solving the problem with only one $f_{i}$ as objective function. Table 1 shows results of this operation. Then a new problem is solved whose objective function is (18) and note that parameter of $P$ is considered equal to 1 in this paper. Finally, new problem by using of table 1 data and equation (18) is constructed as equation (19).

As mentioned in the previous section, for obtain a Pareto optimal set with 10 members, we need to 10 preference vectors. These vectors, which are shown in Table 2, guaranteed a suitable diversity and distribution for Pareto optimal set because they produced based on explained logic in the previous section. This issue also is depicted in Figure 2.

In this work we used CPLEX solver of GAMS 22.2 software for optimizing of the constructed MODM model. Meanwhile, note that this model is executed on a Pentium Core Duo processor with $4 \mathrm{~GB}$ of RAM and $2.2 \mathrm{GHz}$ of CPU.

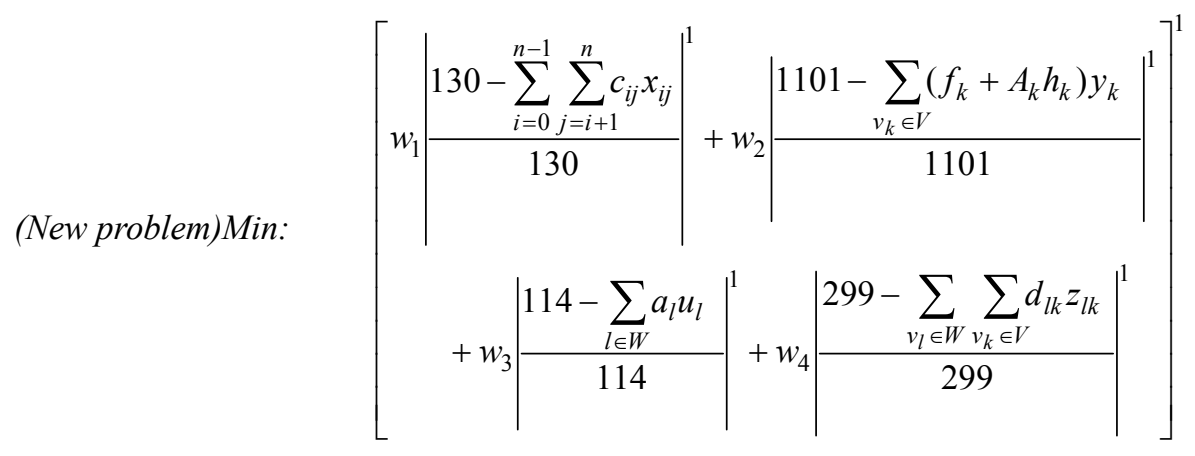

Subject to: (5),(6),(7),(8),(9),(10),(11),(12),(13),(14),(15),(16),(17)

Results for our instance which are Pareto optimal solutions are depicted in Table 3. The column headings are defined for this table as follows:

$T L$ : Tour length

$T C$ : Total clinic building cost

$B C$ : Backup coverage (first service level indicator)

$A L$ : Allocation cost (second service level indicator)

$V$ : Visited cities set

$S T$ : Necessary time for stop in each city (one time unit for any allocated demand point)

$N B C$ : Number of demand point with backup covering

$P B C$ : Percent of demand point with backup covering

For example, number 3 case will be illustrated. Preference vector in this case is $(0.25,0.25,0.25,0.25)$, so all of objective functions importance is same. If objective function values in this row of table 3 compare with the optimal values in table 1, one can see as well as all of objective functions have gotten value with a distance form optimal value and this event is completely natural in MODM problems. Because solutions in such problems optimize all objective functions simultaneously and not only one objective functions. In this case tour length, 
total fixed and variable clinic building cost, total utility of backup coverage and allocation cost are equal to: 149 , $1267,87,317$ respectively. Health care teams should pass from cities 1, 3, 4, 5, 6, 7 and 9. Numbers of demand points that are allocated to these cities consist: $2,3,3,5,2,4$ and 3 respectively and health care teams should divide their time between visited cities based on these allocations and for example should be consider 2 time units for stop in the city 1 . Transmission of this path redounds to impart 15 demand points from backup coverage and so 68 percent of total demand points use of this vantage.

Moreover, trade-off between obtained Pareto optimal solutions is visible truly. We consider two solutions which have almost inverse preference vectors, mean solutions of 2 and 6 , for example. In second solution, tour length and backup covering is more importance, while in sixth solution allocation and clinic building cost is premier. Below comparisons shows exchange between these solutions truly.

$$
\begin{gathered}
T L(2)=161<T L(6)=253 \\
T C(2)=1666>T C(6)=1259 \\
B C(2)=109>B C(6)=84 \\
A L(2)=355>A L(6)=310
\end{gathered}
$$

It is clear that solution of 2 has better conditions in tour length and backup covering, while solution of 6 in allocation and clinic building cost has better position. Computations results prove that this new multi-objective model is more comprehensive and practical with respect to the CTP.

\section{Conclusion and future research}

In this work we have studied a generalization of the covering tour problem, namely allocated maximal backup covering tour problem (AMBCTP). The AMBCTP is a multi-objective problem and aims to optimizing four objectives at a same time: (i) the minimization of the tour length, (ii) the maximization of the backup coverage, (iii) the minimization of the allocation cost and (iv) the minimization of the total clinic building cost. Finally a numerical example illustrated the proposed model. Results showed that the AMBCTP is a comprehensive and practical problem. Development of our model in capacitated form, considering the problem in a fuzzy or stochastic space for this problem can be discussed as future researches.

\section{References}

Araz, C., Selim, H., \& Ozkarahan, I. (2007). A fuzzy multi-objective covering-based vehicle location model for emergency services. Computers \& Operations Research, 34, 705-726. http://dx.doi.org/10.1016/j.cor.2005.03.021

Chankong, V., and Haimes, Y. (1983). Multiobjective Decision Making: Theory and Methodology. New York: North-Holland.

Chen, P-C., \& Huang, C-M. (2004). Biobjective Power Dispatch Using Goal-Attainment Method and Adaptive Polynomial Networks. IEEE Transactions on Energy Conversion, Vol. 19, No. 4. http://dx.doi.org/10.1109/TEC.2003.822306

Chen,Y-L., \& Liu, C-C. (1994). Multi-objective VAR planning using the goal-attainment method. IEE hoc.iener. Transm. Distrib. Vol. 141, No. 3.
Cooper,
L. (1963).
Location-allocation
problems.
Oper.Res11,
pp.331-343. http://dx.doi.org/10.1287/opre.11.3.331

Current, J. R., and Schilling, D. A. (1989). The Covering Salesman Problem. Transp. Sci. 23, 208-213. http://dx.doi.org/10.1287/trsc.23.3.208

Current, J. R., and Schilling, D. A. (1994). The Median Tour and Maximal Covering Problems. European J. Opnl. Res. 73, 114-126. http://dx.doi.org/10.1016/0377-2217(94)90149-X

Current, J.R. (1981). Multi-objective Design of Transportation Networks. Ph.D. Thesis, Department of Geography and Environmental Engineering. The Johns Hopkins University.

Deb, K. (2001). Multi-objective Optimization using Evolutionary Algorithms, Wiley-Interscience Series in Systems and Optimization, John Wiley \& Sons.

Foord, F. (1995). Evaluation of the mobile health care service in West Kiang district. World Health Statistics Quarterly, 48, 18-22.

Gembicki, F.W., and Haimes, Y. Y. (1975). Approach to performance and sensitivity multiobjective optimization: The goal attainment method, IEEE Trans. Automat. Control, vol. 20, pp. 769-771. 
http://dx.doi.org/10.1109/TAC.1975.1101105

Gendreau, M., Laporte, G., \& Semet, F. (1997). The covering tour problem. Operations Research, 45, 568-576. http://dx.doi.org/10.1287/opre.45.4.568

Hachicha, M., Hodgson, M.J., Laporte, G., \& Semet, F. (2000). Heuristics for the multi-vehicle covering tour problem. Computers \& Operations Research, 27, 29-42. http://dx.doi.org/10.1016/S0305-0548(99)00006-4

Hodgson, M.J., Laporte, G., and Semet, F. (1998). A covering tour model for planning mobile health care facilities in Suhum district, Ghana. Journal of Regional Science, 38, 621-638. http://dx.doi.org/10.1111/0022-4146.00113

Hogan, K., \& ReVelle, C. (1986). Concepts and applications of backup coverage. Management Science. 32, 1434-1444. http://dx.doi.org/10.1287/mnsc.32.11.1434

Islam, S. (2008). Multi-objective marketing planning inventory model: A geometric programming approach. Applied Mathematics and Computation, 238-246. http://dx.doi.org/10.1016/j.amc.2008.07.037

Jozefowiez, N., Semet, F., and Talbi, E. (2007). The bi-objective covering tour problem. Computers \& Operations Research, 34, 1929-1942. http://dx.doi.org/10.1016/j.cor.2005.07.022

Low C., Wu, T-H., \& Hsu, C-M. (2005). Mathematical modelling of multi-objective job shop scheduling with dependent setups and re-entrant operations. Springer-Verlag London, 27, 181-189.

Maniezzo, V., Baldacci, R., Boschetti, M., and Zamboni, M. (1999). Scatter search methods for the covering tour problem. Technical Report, Science dell'Informazione, University of Bologna, Italy.

Motta, L., Ochi, LS., \& Martinhon, C. (2001). Grasp metaheuristics for the generalized covering tour problem. In MIC'2001-fourth metaheuristics international conference. Porto, Portugal. p. 387-391.

Motta, L., Ochi, LS., \& Nogueira, L. T. (2010). Improving performance of algorithms for the covering tour problem by applying reduction rules.

Oppong, JR., \& Hodgson, MJ. (1994). Spatial accessibility to health care facilities in Suhum district, Ghana. The Professional. Geographer, 46, 199-209. http://dx.doi.org/10.1111/j.0033-0124.1994.00199.x

Salukvadze, M. E. (1974). On the existence of solution in problems of optimization under vector valued criteria. Journal of Optimization Theory and Applications, 12(2), 203-217. http://dx.doi.org/10.1007/BF00935540

Swaddiwudhipong, W., Chaovakiratipong, C., Nguntra, P., Lerdlukanavonge, P., \& Koonchote, S. (1995). Effect of a mobile unit on changes in knowledge and use of cervical cancer screening among rural Thai women. International Journal of Epidemiology, 24, 493-498. http://dx.doi.org/10.1093/ije/24.3.493

Table 1. Optimal value of objective functions

\begin{tabular}{|c|c|c|c|c|}
\hline $\begin{array}{c}\text { Objective } \\
\text { function }\end{array}$ & $\sum_{i=0}^{n-1} \sum_{j=i+1}^{n} c_{i j} x_{i j}$ & $\sum_{v_{k} \in V}\left(f_{k}+A_{k} h_{k}\right) y_{k}$ & $\sum_{l \in W} a_{l} u_{l}$ & $\sum_{v_{l} \in W} \sum_{v_{k} \in V} d_{l k} z_{l k}$ \\
\hline Optimal value & 130 & 1101 & 114 & 299 \\
\hline
\end{tabular}


Table 2. Preference vectors

\begin{tabular}{|c|c|c|c|c|}
\hline Vector Number & $w_{1}$ & $w_{2}$ & $w_{3}$ & $w_{4}$ \\
\hline $\mathbf{1}$ & 0.1 & 0.1 & 0.2 & 0.6 \\
\hline $\mathbf{2}$ & 0.2 & 0 & 0.8 & 0.0 \\
\hline $\mathbf{3}$ & 0.25 & 0.25 & 0.25 & 0.25 \\
\hline $\mathbf{4}$ & 0.4 & 0.2 & 0.0 & 0.4 \\
\hline $\mathbf{5}$ & 0.5 & 0.1 & 0.4 & 0.0 \\
\hline $\mathbf{6}$ & 0.0 & 0.4 & 0.0 & 0.6 \\
\hline $\mathbf{7}$ & 0.7 & 0.2 & 0.05 & 0.05 \\
\hline $\mathbf{8}$ & 0.75 & 0.1 & 0.05 & 0.1 \\
\hline $\mathbf{9}$ & 0.1 & 0.2 & 0.6 & 0.1 \\
\hline $\mathbf{1 0}$ & 0.0 & 0.7 & 0.3 & 0.0 \\
\hline
\end{tabular}

Table 3. Pareto optimal set with 10 members

\begin{tabular}{|c|c|c|c|c|c|c|c|c|}
\hline $\begin{array}{l}\text { Solution } \\
\text { Number }\end{array}$ & $T L$ & $T C$ & $B C$ & $A L$ & $V$ & $S T^{*}$ & $N B C$ & $P B C(\%)$ \\
\hline 1 & 161 & 1537 & 109 & 307 & $1,2,3,4,5,6,7,9$ & $2,2,2,3,4,3,4,2$ & 18 & 81.8 \\
\hline 2 & 161 & 1666 & 109 & 355 & $1,2,3,4,5,6,7,8,9$ & $0,3,2,3,1,3,2,4,4$ & 18 & 81.8 \\
\hline 3 & 149 & 1267 & 87 & 317 & $1,3,4,5,6,7,9$ & $2,3,3,5,2,4,3$ & 15 & 68 \\
\hline 4 & 137 & 1143 & 34 & 326 & $1,2,3,4,5,6,7$ & $4,2,3,4,5,4$ & 6 & 27.2 \\
\hline 5 & 161 & 1481 & 109 & 384 & $1,2,3,4,5,6,7,9$ & $2,0,5,6,4,1,4,0$ & 18 & 81.8 \\
\hline 6 & 253 & 1219 & 84 & 310 & $1,3,5,6,7,9,10$ & $3,2,5,3,4,3,2$ & 15 & 68 \\
\hline 7 & 137 & 1111 & 65 & 339 & $1,3,4,5,6,7$ & $2,7,2,5,2,4$ & 12 & 54.5 \\
\hline 8 & 137 & 1124 & 65 & 331 & $1,3,4,5,6,7$ & $3,5,2,4,3,4$ & 12 & 54.5 \\
\hline 9 & 161 & 1503 & 109 & 316 & $1,2,3,4,5,6,7,9$ & $1,1,6,2,5,1,4,2$ & 18 & 81.8 \\
\hline 10 & 268 & 1186 & 84 & 396 & $1,3,5,6,7,9,10$ & $1,9,4,1,4,2,1$ & 15 & 68 \\
\hline $\begin{array}{l}\text { Total } \\
\text { time: }\end{array}$ & $147 r$ & S & & & & & & \\
\hline
\end{tabular}

*Order of elements in this column is same order of $V$ column. 

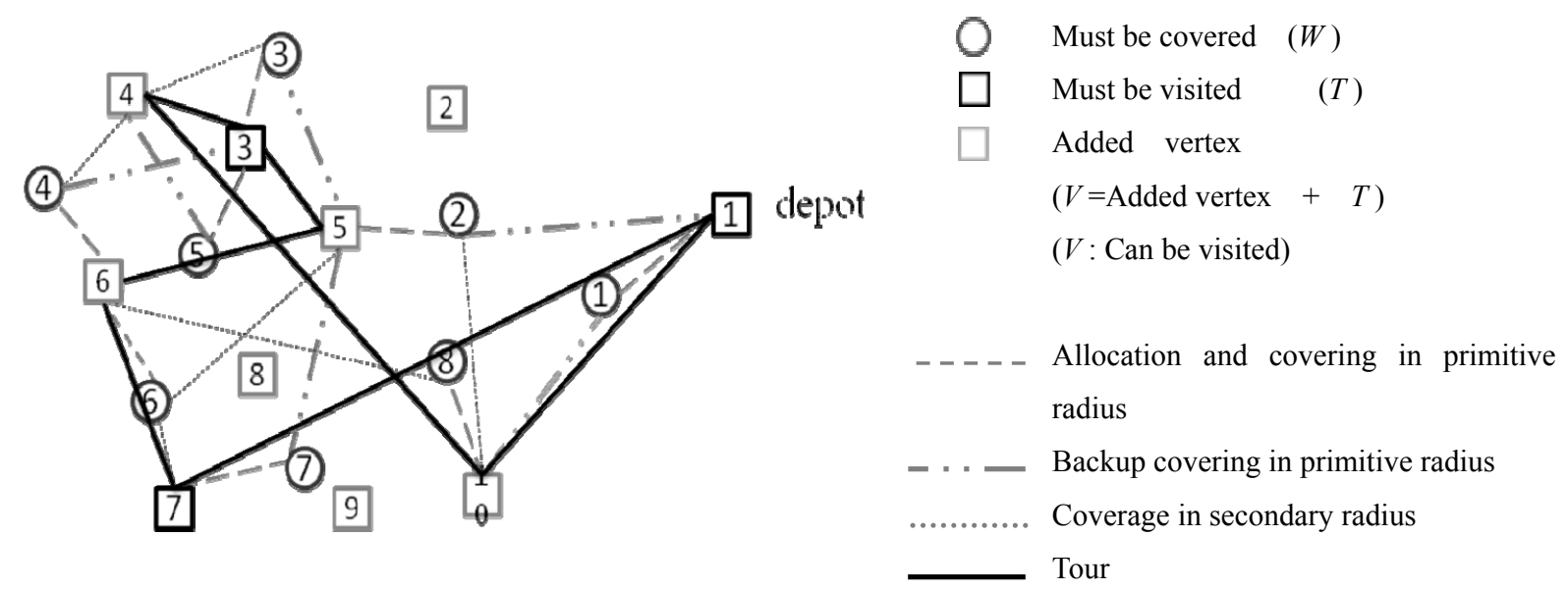

Figure 1. An example of a solution for the AMBCTP

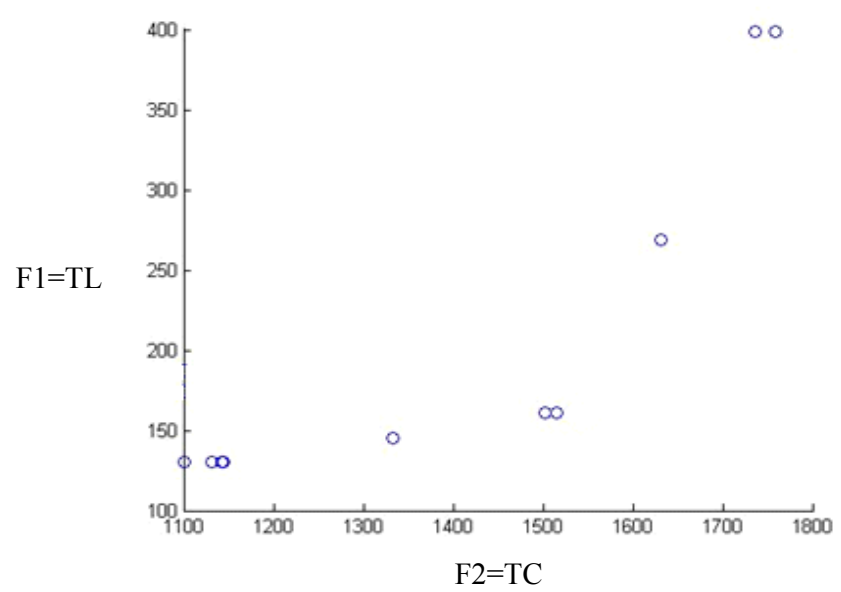

Figure 2. Display distribution and diversity for Pareto optimal set in case of first and second objective 